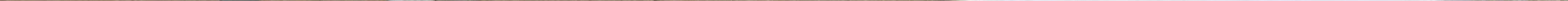



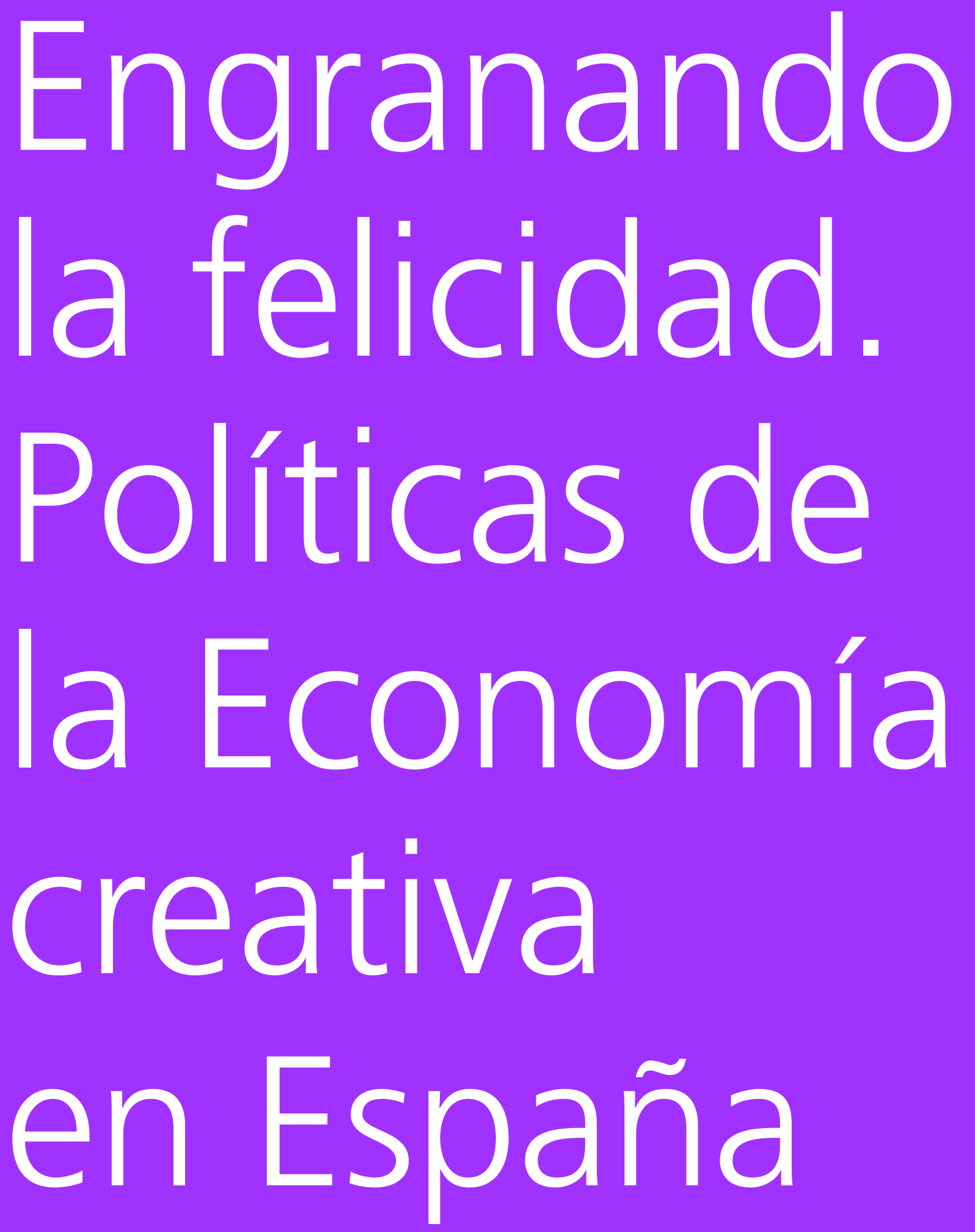

Victoria Clemente

Clemente, V. (2015). Engranando la felicidad. Políticas de la Economía creativa en España, Economía Creativa. (04) otoño-invierno 2015, México: pp. 31-61. 


\section{Engranando la felicidad. Políticas de la Economía creativa en España}

Victoria Clemente ${ }^{1}$

\section{Resumen}

En las últimas décadas hemos presenciado una serie de cambios graduales en nuestra economía y sociedad que parecen haber traído una nueva forma de trabajar y de vivir. A este suceso le denominamos fenómeno creativo porque es precisamente la creatividad la que se está convirtiendo en un importante factor de crecimiento económico. A pesar de ser evidentes los beneficios de su aplicación a los múltiples sectores de desarrollo, parece que las políticas públicas españolas aún no se sustentan, en su mayoría, sobre bases creativas. El presente estudio busca analizar por un lado, el beneficio de la aplicación de la creatividad en el hombre y en el territorio, y por otro, la repercusión que el fenómeno creativo está teniendo en las retóricas que dan sentido a las políticas públicas españolas. Finalmente, el artículo está enfocado a demostrar que la creatividad es una llave valiosa para el crecimiento económico e impulso del potencial humano en general.

Palabras clave | Economía Creativa, valor, políticas públicas, desarrollo, local.

1 victoria@icdea.es, iCdea 
ENGRANANDO LA FELICIDAD.

POLÍTICAS DE LA ECONOMÍA CREATIVA EN ESPAÑA

\begin{abstract}
In the last decades we have witnessed ongoing changes in economy and socie-ty that seem to have brought as result, new working methods and lifestyles. This event is what we call the creative phenomenon, because it is precisely creativity what is becoming an important factor for economic growth. Despite the obvious benefits of its application on different developing sectors, it appears that the Spanish public policies are not supported yet, mostly on creative bases.

This study seeks to analyze on one side, the benefit of the application of crea-tivity in man and in its territory, and in the other, the impact that the creative phenomenon is having on the rhetoric that gives meaning to the Spanish public policies. Finally, the article hopes to demonstrate that creativity is in general an essential key on economic growth and promotion of human potential.
\end{abstract}

Keywords | Creative Economy, value, public policies, development, local. 
ENGRANANDO LA FELICIDAD.

POLÍTICAS DE LA ECONOMÍA CREATIVA EN ESPAÑA

Un buen día aparecerá en el aire una especie de Arco Iris inmenso y volveremos a tener poesía, música, pintura y escultura verdaderas, limpias, desnudas, sin colgajos adheridos, sin ingeniosidades pegadas, sin sustos, sin sorpresas, sin modas más o menos baratas, sin modernidades... (Gaya, 1996, 57).

Las ideas recogidas en este artículo no tienen pretensión alguna de formar un texto orgánico y hermético sino que de forma sencilla, intentan reflejar una realidad que, como espectadora profesional, he podido percibir a mi alrededor en la última década. Recogidos, en un contenedor abierto, pensamientos coleccionados durante los últimos años de investigación sobre economía creativa, he intentado unirlos a la compleja y parcial realidad próxima a mi experiencia. Desde esta idea, este documento recoge lo que, a mi juicio, en España como en otros países, está siendo un camino hacia un nuevo modo de entender el desarrollo económico y social, un brasero que no sólo parece no apagarse, sino ser más indómito que nunca. Se trata, por tanto, de un artículo para subrayar la importancia de aquellos valores que tanto cuesta medir, así como para resaltar el carácter fundamental de las inversiones que generan retornos no inmediatos y no están centrados únicamente en el aporte económico.

Decía el pintor y escritor Ramón Gaya en sus anotaciones que "volver a lo antiguo será ya un progreso" (Gaya, 2010, 407); resulta curioso cómo el creador nos anima a volver al origen, a comenzar de nuevo para reparar -quizá- lo que parece haberse planteado mal desde su inicio. Y con esta idea conectamos directamente el proceso vivo de la economía creativa: con la autenticidad, con lo esencial, con lo sencillo, con amar lo que se hace... porque todo parece nacer de ahí y, a la vez, dirigirse al mismo lugar.

Siguiendo la afirmación de Gaya referida al proceso de creación podríamos pensar que no se hace necesario un nuevo comienzo, sino más bien, una tarea de revisión acerca de lo que hay creado hasta ahora y como consecuencia de ésta, un análisis de las políticas públicas que mueven a las sociedades.

A priori, puede resultar atrevido enlazar los procesos creativos con el desarrollo de políticas públicas, pero veremos, a través de las referencias encontradas, cómo ambos conceptos están unidos a través de un proceso de retroalimentación. 
ENGRANANDO LA FELICIDAD.

POLÍTICAS DE LA ECONOMÍA CREATIVA EN ESPAÑA

Uno de los últimos estudios sobre industrias creativas en España, el de los autores Boix y Lazzeretti (2012), resalta como idea clave que la Economía creativa se ha convertido en un factor de desarrollo. En él, afirman que la Economía creativa es importante porque genera más del $6 \%$ del producto interior bruto mundial. En la misma línea, también Howkins (2013) apunta que la creatividad parece haberse convertido en la base de la innovación, además, el autor resalta, que con su uso el modelo social está siendo distinto.

Y en esta misma dirección, es donde enlaza la activación de la Economía creativa con la idea de nuevos modelos de ciudades y ciudadanos. Sin suponer una ruptura con los anteriores procesos, parece que el camino está en seguir forjando la retórica de las políticas públicas sustentada, principalmente, en las tendencias sociales de crecimiento, en lo que sucede en los contextos donde se está desarrollando la creatividad, es decir, desde la aplicación de la creatividad del ciudadano para la ciudadanía.

De forma paralela al desarrollo cultural de las ciudades, emergen entornos de actividad creativa que generan nuevas formas de pensamiento y que tienen mucho que ver con el discurso que buscan las políticas de desarrollo. El profesor italiano Nuccio Ordine recoge, en La utilidad de lo inútil (2013), citas y pensamientos desde la antigüedad que ponen nuevamente de manifiesto la gran importancia que la cultura tiene para el desarrollo del hombre:

La utilidad de los saberes inútiles se contrapone radicalmente a la utilidad dominante que, en nombre de un exclusivo interés económico, mata de forma progresiva la memoria del pasado, las disciplinas humanísticas, las lenguas clásicas, la enseñanza, la libre investigación, la fantasía, el arte, el pensamiento crítico y el horizonte civil que debería inspirar toda la actividad humana. $(2013,11)$.

Lejos del interés económico del que habla Ordine se encuentra parte de la esencia de la Economía creativa, una economía en la que sus actividades están basadas en principios creativos y en la generación de ideas. Se trata de un modelo de economía vinculado a un sector en el que los parámetros para medir su éxito están ligados a la identidad y a otros valores del ser humano en primera instancia, dejando de ser el beneficio económico la premisa más importante. 
ENGRANANDO LA FELICIDAD.

POLÍTICAS DE LA ECONOMÍA CREATIVA EN ESPAÑA

En la última década, el significado de valor parece adquirir importancia socialmente. ¿Qué es lo vale en nuestra vida? Intentando hacer una rápida aproximación, podríamos decir que hasta hace poco, el mercado fijaba los precios y, casi de forma automática, eran estos los encargados de dar el valor a las cosas, a los productos y servicios. Aunque este proceso se siga dando, de un tiempo a esta parte, dentro del sector creativo ${ }^{2}$ no parece estar tan clara la unión de precio y valor, una asociación atribuida que, en realidad, quizá nunca debió existir.

Tras la crisis del sistema económico, buscando incesantemente una recuperación de los modelos de bienestar, el ser humano, impulsado también por una búsqueda de sí mismo y de crecimiento de su comunidad, comienza a valorar en mayor medida "lo singular, lo simbólico y lo intangible"; tres pilares de la Economía creativa según Fonseca $(2008,9)$.

Volviendo a la publicación de Ordine (2013), seleccionamos algunas de sus anotaciones que señalan la cuestión que analizamos. El autor indica en su manifiesto que ya Stevenson, en La isla del tesoro, en sintonía con esta observación, manifestaba que era mucho más valioso el ser que el tener o Shakespeare, que ya imaginaba un reino inmune a la fiebre del beneficio (38). En este sentido entendemos que, desde siempre, la necesidad de ser ha estado intrínseca en el ser humano, y que, de algún modo, parece haberse visto enturbiada por un sistema económico que, en algún momento, consiguió que mirásemos desde la misma óptica el concepto de valor y el de precio.

Y precisamente en esa búsqueda del ser, en esa necesidad de encontrarse el hombre, se halla quizá uno de los principios de la creatividad, aplicada en esta ocasión a cualquier actividad que implique el desarrollo de un trabajo. Quizá también la necesidad de reinventarse para sobrevivir, generada por la crisis económica, sea otra de las principales cuestiones que nos impulsen hacia este nuevo modelo. En cualquier caso, lo que nos resulta incuestionable es el fenómeno creativo y las consecuencias que está teniendo en el PIB español y en el desarrollo local de los territorios. ${ }^{3}$

\footnotetext{
2 Hacemos alusión a la industria creativa y por ende, a la Economía Creativa.
}

3 Según la Conferencia de Naciones Unidas para el Comercio y el Desarrollo (UNCTAD), entre el 2002 y el 2011, las exportaciones de Bienes y Servicios Creativos crecieron un $134 \%$. 
ENGRANANDO LA FELICIDAD.

POLÍTICAS DE LA ECONOMÍA CREATIVA EN ESPAÑA

El filósofo Karl Marx (Sossa, 2010), quien también incursionó en los campos de la ciencia política, la sociología y la economía, acuñó alienación como concepto psicológico y sociológico. Consideraba que el trabajador, desde el punto de vista capitalista, ya no era una persona en sí misma, sino una mano de obra que podía representarse en su equivalente económico. El trabajador era, por tanto, una determinada cantidad, utilizable como producción para la multiplicación del dinero.

En una línea totalmente opuesta al concepto de alienación podríamos situar el desarrollo de la Economía creativa donde el hombre comienza a verse inmerso en un fenómeno contrario al obtenido desde el sistema capitalista. El trabajador en sí añade un valor a su mano de obra, comienza a dejar de ser una cantidad monetaria y su pensamiento y creatividad parecen convertirse en lo importante y en lo real, en lo verdaderamente valioso. El trabajo mecánico resta importancia y se complementa por ideas de originalidad que dan sentido social al unirlas con las ideas del igual.

Desde esta perspectiva, podríamos pensar que contrario al concepto de alienación, en el que la estructura social capitalista generaba distorsiones en la naturaleza humana, se reafirma una nueva forma de entender el trabajo del hombre, un modelo que no solo no parece desestructurar, sino dar sentido al trabajo creador. Esta teoría podría estar haciendo que el hombre se considere una figura valiosa dentro de un sistema económico, que ya no sólo parece enfocar hacia el precio de sus productos y servicios, sino que, cada vez más, se aproxima a hacer visible su valor, humanizando el trabajo.

A su vez, también el filósofo indicaba que el trabajo desempeñado por el hombre surgía de la necesidad de relacionarse socialmente, naciendo así, de esta relación laboral, lo que él llamaba una conciencia social colectiva, un estado que quizá podría ayudar a desarrollarse a los territorios. Esta idea parece aproximarnos más aún al objeto de análisis de este artículo: la cooperación de los hombres en la creación y desarrollo de sus entornos o territorios. Y es que una de las características que dan fuerza al nacimiento de la Economía creativa es la comunicación del hombre con su entorno, con el territorio donde habita. Se trata también de una cuestión fundamental para el investigador Richard Florida (2012) que destaca la importancia del territorio como la unidad de organización más importante para el desarrollo social y económico. 
ENGRANANDO LA FELICIDAD.

POLÍTICAS DE LA ECONOMÍA CREATIVA EN ESPAÑA

De esta forma, encontramos relación entre el uso, cada vez mayor, de la creatividad humana aplicada al trabajo del hombre con el desarrollo de los territorios. Puede que incluso hablando de economía creativa, estemos abordando una de las necesidades más básicas del ser humano: la de poner toda su creatividad al servicio de la sociedad que habita para hacer de ella un lugar mejor.

¿Le resulta por tanto evidente a la humanidad la necesidad de liberación de todos los condicionamientos establecidos? No quiere decirse con ello que sea necesaria una rebelión, pero sí el hecho preciso de insistir en el empeño de generar valor a partir de un cambio y de nuevos modelos colaborativos sociales y, sobre todo, desde la observación de los mismos. Ahora veremos porqué.

\section{Lo valioso y la Economía Creativa}

Resulta un hecho obvio que una misma escena pueda asumir significados distintos en un mismo contexto. Dos conceptos semejantes de emprendimiento o negocio, dos obras de arte, dos poemas... pueden ser totalmente diferentes, si uno de ellos nace, en su base más pura, desde la libre creatividad del ser humano. En este sentido y más próximos al concepto de valor, ahondaremos en la inseparable relación de este con la emergente Economía creativa.

Durante la última década, diferentes expertos de varios continentes como Ana Carla Fonseca, Edna dos Santos- Duisenberg, Yudhishthir Raj, Máté Kovács, Andrea M. Davis (Fonseca, 2008)... han puesto de manifiesto resultados que prueban la existencia de un sector productivo, valioso y potente, que tiene sus bases en la creatividad y está formado -principalmentepor actividades y recursos culturales y creativos y con un gran potencial para el desarrollo.

También algunas contribuciones clave han sido las ediciones de 2008, 2010 y 2013 del Informe sobre la Economía creativa, elaboradas por la Conferencia de las Naciones Unidas sobre Comercio y Desarrollo (UNCTAD) y las del Programa de las Naciones Unidas para el 
ENGRANANDO LA FELICIDAD.

POLÍTICAS DE LA ECONOMÍA CREATIVA EN ESPAÑA

Desarrollo (PNUD). Ambas publicaciones demuestran que la Economía creativa no sólo es uno de los sectores que más rápido está creciendo dentro de lo que sería el sistema económico mundial, sino que, además, se ha convertido en un proceso transformador en términos de generación de recursos tangibles e intangibles, de creación de empleo, de aumento en los ingresos de las exportaciones... en definitiva, de desarrollo.

Una de las conclusiones más rotundas de estas observaciones es la tendencia en la aplicación de mecanismos que parecen ser diferentes a los que existían hasta ahora. Se trata de una evolución hacia el uso de nuevas herramientas como la creación de engranajes participativos fomentadores de la implicación social activa en procesos de elaboración de políticas públicas de desarrollo.

A su vez, algunos informes también desvelan haber identificado factores fundamentales de éxito, que van desde el desarrollo de las capacidades locales hasta la gestión propia de los bienes por parte de los habitantes, pasando de este modo por la facilitación de las conexiones y los flujos transnacionales.

Hasta ahí todo nos puede parecer lógico: la creatividad desarrolla territorios a través de nuevos modelos de emprendimiento, de nuevas políticas enfocadas a programas participativos, al ciudadano... y ésta, a su vez, consigue activar un tejido empresarial urbano que potencia a las ciudades mediante la activación del mercado y generando más y renovadas políticas públicas. Pero, si es tan sencillo, ¿por qué no se está aplicando en las políticas de desarrollo de muchas ciudades?, ¿por qué la Economía creativa no es la base del desarrollo de la mayoría de políticas públicas? Quizás, todo deba darse en su contexto y en su momento; de lo contrario, restaría sentido.

Valorando el potencial que tiene la Economía creativa y a pesar de reconocer la tendencia que se afirma con anterioridad, uno de los últimos estudios sobre cultura, creatividad y economía urbana en España, realizado por la Universidad del País Vasco (Méndez et al, 2012), a alerta de la escasa contrastación empírica y analítica en torno a la aportación y procesos impulsores de las industrias culturales y creativas. Resulta cuestionable, según el estudio, la acogida de la Economía creativa y su incorporación a la retórica de las políticas públicas. 
ENGRANANDO LA FELICIDAD.

POLÍTICAS DE LA ECONOMÍA CREATIVA EN ESPAÑA

Por lo tanto, sin perder de vista la escasez de resultados tangibles hasta el momento, partimos de la idea de las grandes ventajas que está suponiendo para el desarrollo la potenciación de la Economía creativa. No obstante, no debemos olvidar una estructura tan firme como la que resalta el profesor Ordine en cuanto a la importancia que tiene la cultura para el desarrollo (2013), pues, de algún modo, ésta se conforma de actos totalmente puros de gran carácter simbólico y singular, exportando al resto de campos socioeconómicos un conjunto de valores en sintonía con la tendencia de desarrollo sostenible actual.

Aún así, y más allá de los escasos resultados, ${ }^{4}$ estas teorías sobre Economía creativa pueden tomarse al menos como un termómetro que mida las transformaciones en el seno de lo social, cultural y económico. Un ejemplo sería el aumento en escala del sentido y del valor, acciones que van adquiriendo un formato político-económico del Estado del Bienestar y desarrollan factores de intervención local y específicos a los territorios el conocimiento, las relaciones sociales, la creatividad, las redes de confianza... en definitiva, características que están convirtiendo a cada espacio en singular y diferenciado. Un ejemplo de ello sería el proceso de crecimiento del céntrico barrio de Lavapiés en Madrid. ${ }^{5}$

Asimismo, conscientes de las conclusiones de los estudios consultados, pensamos que, por el momento, se debería partir de un proceso lento basado -principalmente- en la observación, acogiendo el fenómeno creativo desde un equilibro que, por un lado nos permita ir evaluando sus beneficios a corto y medio plazo (tangibles e intangibles) y, por otro, ir incorporando lentamente su filosofía en la retórica de los programas de futuro.

${ }^{4}$ Al tratarse de un concepto joven no son abundantes los resultados que muestran el potencial del sector así como las carencias que pueda presentar.
${ }^{5}$ En Cañedo 2006 puede encontrarse un desarrollo de algunos aspectos que tan sólo son menciones aquí, así como un análisis de los distintos perfiles de vecinos de Lavapiés y de su interacción en relación al problema de la construcción del barrio como lugar urbano. 


\section{Vale más dar que tener}

Desde una concepción más romántica, en las últimas décadas, también el fenómeno creativo ha estado en el pensamiento de numerosos autores, filósofos y antropólogos. Hemos podido comprobar cómo pensadores hablan de la necesidad de las cosas inútiles para el desarrollo de la sociedad (Ordine, 2013). Pensamientos a lo largo de la historia coinciden en resaltar el hecho de cómo la creatividad del hombre, el proceso de creación en sí, ha hecho y hacen más bello el camino.

Esta afirmación nos sitúa nuevamente en el manifiesto del profesor italiano, que en definitiva, expone de modo rotundo cómo a través del tiempo la cultura y la creatividad han sido útiles para el desarrollo de la sociedad.

Existen saberes que son fines por sí mismos y que- precisamente por su naturaleza gratuita y desinteresada, alejada de todo vínculo práctico y comercial- pueden ejercer un papel fundamental en el cultivo del espíritu y en el desarrollo civil y cultural de la humanidad. En este contexto, considero útil todo aquello que nos ayuda a hacernos mejores (Ordine, 2013, 9).

El autor, haciendo especial hincapié en la utilidad de lo que nos hace mejores, sintoniza con la tendencia de la aplicación de la creatividad del ser humano y llegados a este punto cabe preguntarnos: ¿qué necesita la humanidad para su desarrollo civil y cultural? Podríamos pensar que lo necesario para el hombre es todo lo que le resulta útil, pero ¿qué es útil? Puede que, hasta hace unos años, el ser humano adoptara la idea de que útil era todo aquello que generaba un beneficio económico e incluso, se podía pensar que la única recompensa de un intercambio o transacción era la monetaria. Pero si ahondamos en cómo ha ido transcurriendo su crecimiento así como el de la sociedad en sí, veremos que la tendencia actual nos conduce -obligados o no- a valorar otros muchos aspectos además del monetario, haciendo que adquieran valor factores que pasaban desapercibidos. El autor, basado en este pensamiento, insiste en la idea de hacernos mejores personas a través de todo aquello que nos sea útil, conlleve o no finalmente a un beneficio económico. 
ENGRANANDO LA FELICIDAD.

POLÍTICAS DE LA ECONOMÍA CREATIVA EN ESPAÑA

Una de las bases sobre las que se sustenta la Economía creativa parece ser la de poner en alza aspectos del hombre y de su trabajo que, hasta hace poco, podrían haber sido considerados como inútiles o, simplemente, no haberse tenido en cuenta. Y no sólo estamos pensando en la cultura, que mediante ella todo tiene su principio, sino en otro tipo de saberes y conocimientos que hasta hoy no se hubieran considerado útiles para potenciar el desarrollo económico y social de un territorio. Fonseca prevé el futuro próximo de la Economía creativa señalando lo siguiente:

La economía creativa se despliega en dos enfoques complementarios. El primero tiene como base el reconocimiento de la creatividad y, por lo tanto, del capital humano para el fomento de una integración de objetivos sociales, culturales y económicos, mediante un modelo de desarrollo global postindustrial excluyente, y por lo tanto, insostenible (Fonseca, 2008, 27).

En este sentido, nos es inevitable pensar que el remedio de la dura austeridad, aplicado a la sociedad en los últimos años, no sólo no ha hecho que no mejore la situación económica del sistema, sino que, observando las diferentes teorías económicas acerca de la situación, podemos tender a pensar que, en vez de mejorarla ha conseguido debilitarla más. Cuestionamos en ese sentido si las políticas públicas de desarrollo se han resquebrajado perdiendo su lógica y lamentablemente, todo parece apuntar hacia una desestructura. Aunque como opción contraria y paralela a la falta de estrategia, mientras tanto, mientras se balancean en idas y venidas las propuestas de mejora que tan sólo parecen ocultar sin sanar raíces, paralelamente, un nuevo modo de hacer, impulsado desde el estómago del hombre, nace con fuerza para replantear el camino creado hasta ahora.

De este modo, podríamos pensar que la necesidad de mejorar el modelo de desarrollo sumada a un mayor aporte de valor, pueden haberse convertido en otro de los factores detonantes de la potenciación de la Economía creativa. 


\section{Creación y economía: Trabajadores felices}

Mi definición del núcleo de la clase creativa incluye a las personas que se dedican a la ciencia y la ingeniería, a la arquitectura y al diseño, a la educación, al arte, y a la música y el espectáculo, y cuya función es generar nuevas ideas, nueva tecnología y/o nuevos contenidos creativos. Alrededor de este núcleo, la clase creativa también abarca a un grupo más amplio de profesionales creativos, en el mundo de la empresa y de las finanzas, en el ámbito legal y en el sanitario, y en otros campos relacionados. (Florida, 2010, 47).

La clasificación de Florida nos sitúa ante un entremezclado y amplio abanico de trabajos y estilos de vida, los cuales podríamos interpretar -siguiendo la teoría del autor- como el futuro de la reconstrucción, la regeneración y como motor económico de las ciudades.

Puede que nos resulte remoto el hecho de cómo la creatividad se ha convertido en un factor fundamental para el emprendimiento o para el desempeño de cualquier actividad, así como también está resultando relevante su uso para el desarrollo de las ciudades. En cualquier caso, vemos que el proceso de creación está es cada vez más latente. También su vínculo directo con la economía ha generado algunas dudas, pero poco a poco, con los hechos ya existentes, ${ }^{6}$ muchos países se han puesto en marcha redirigiendo sus enfoques de crecimiento desde este concepto. Gracias a programas de financiación europea como Europa Creativa, ${ }^{7}$ parece que lentamente, más empresas europeas comienzan a estar envueltas en procesos creativos, pues no se trata tanto de un proceso de financiación, como de información y concienciación ${ }^{8}$. Así, en esa dirección, algunas empresas comienzan a ampliar su visión y a apostar por otros perfiles profesionales más creativos que parecen generar

\footnotetext{
6 Véase el estudio de Boix y Lazzeretti, (2012) Las industrias creativas en España: una panorámica donde se afirma que las industrias creativas son una parte relevante de la economía española: 5,75\% de la producción y el 6,5\% del empleo.
}

\footnotetext{
7 europacreativa.es/

8 Europa potencia y premia los aportes de la creatividad a la sociedad intentando generar una estructura de conocimiento y puesta en común entre sus países miembros.
} 
ENGRANANDO LA FELICIDAD.

POLÍTICAS DE LA ECONOMÍA CREATIVA EN ESPAÑA

resultados diferentes. En el caso español, todo parece ir más lento, pues por el momento, el peso de las industrias creativas resulta inferior al de la media europea (Boix y Lazzeretti, 2012), infiriendo de ello que la economía española aún no es del todo una economía especializada en el uso de la creatividad.

En un sentido más local, en el territorio español, concretamente en las grandes ciudades (Madrid y Barcelona), existen cada vez más, pequeños modelos de negocio basados en contenidos originales y servicios más personalizados. Se tratan de modelos que persiguen la personalización y la recuperación de esencias en los barrios donde están siendo instalados. Señalamos la tendencia de la agrupación social, clusters de creación e intercambio y el trabajo colaborativo.

Richard Florida (2010) se aproxima a esta idea añadiendo que el talento es atraído y retenido por las ciudades-región, aludiendo que no se trata de todas las ciudades-región, sino sólo de algunas específicas. Para el autor, los espacios creativos e innovadores son aquellos capaces de aunar lo que él denomina como las '3T's': tecnología, talento y tolerancia. De este pensamiento podemos entender que las ciudades más competitivas, los sistemas más productivos, los que tienen mayor capacidad para atraer el talento y retenerlo, podrían ser aquellas que ofrecen una mayor calidad de vida, con la que, finalmente, se es más feliz. Por tanto, el autor se podría estar refiriendo a ecosistemas o espacios abiertos, caracterizados quizá por una amplia diversidad cultural y social.

Otro de los estudios que tratan la creatividad y su relación con un desarrollo sostenible es la encuesta State of the Global Workplace ${ }^{10}$ realizada en 142 países. En esta ocasión, los resultados destacan la idea de que los trabajadores felices son tres veces más creativos, 73\% más comprometidos y $31 \%$ más productivos. Además resalta que los colaboradores con emociones positivas desarrollan su pensamiento y esto les permite utilizar nuevos comportamientos que generan resultados diferentes.

\footnotetext{
9 Véase La Red de Industrias Creativas creada en la capital española que se ha convertido un ejemplo de creación y potenciación de economía creativa.

reddeindustriascreativas.com/
}

10 La organización Gallup realiza anualmente un proyecto de investigación a nivel mundial sobre el estado del lugar de trabajo, abarcando encuestas en 142 países durante los años 2011 y 2012. Desde los 90, este estudio ha sido respondido por 25 millones de empleados en 189 países. 
ENGRANANDO LA FELICIDAD.

POLÍTICAS DE LA ECONOMÍA CREATIVA EN ESPAÑA

Desde esta perspectiva, interpretamos que el fenómeno creativo no está solo presente en acciones de emprendimiento o en la creación de servicios y productos en sí, sino que yendo más lejos, parece estar expandiéndose hasta la espina dorsal de las filosofías corporativas empresariales, enlazando también como resultado de ello, con la situación de ser partícipes en las retóricas que construyen las políticas públicas.

\section{¿Se trata de un fenómeno imparable?}

En el artículo sobre Economía creativa y desarrollo urbano, elaborado por los investigadores del CSIC Méndez, Michelini, Prada y Tébar (2012), se afirma la evidencia del interés de los gobiernos europeos por el crecimiento que parece provocar el fenómeno creativo:

A lo largo de la última década, la economía creativa ha recibido una creciente atención desde el punto de vista del desarrollo urbano. Es evidente que, al tratarse de actividades intensivas en conocimiento, con una demanda global creciente y un riesgo relativamente bajo de deslocalización empresarial, presentan gran interés para los gobiernos europeos, que ven en ellas un factor clave para la regeneración y el desarrollo sostenido de las economías urbanas (Méndez et al, 2012, 27).

Pongamos además cifras al camino ya recorrido. Para la UNESCO, las industrias culturales y creativas contribuyen en torno al 3,4\% del PIB mundial, y entre el $2 \%$ y el $6 \%$ del PIB de numerosas economías nacionales. En la Unión Europea ocupan a seis millones de profesionales, que mueven 654.000 millones de euros al año (2,6\% del PIB), con datos de 2010.

En el caso español han representado el 4,2\% del PIB, con una importancia económica superior a la de sectores básicos como la agricultura o la energía. Además, España ocupa 
ENGRANANDO LA FELICIDAD.

POLÍTICAS DE LA ECONOMÍA CREATIVA EN ESPAÑA

el puesto cuarto, en el ranking por países europeos del Priority Sector Report ${ }^{11}$, en base al volumen de empleo, con más de 625,000 trabajadores en el sector, y el veinte según el peso del empleo cultural sobre el total de la fuerza de trabajo, con un valor de 4,0\%. Sólo en Madrid trabajan más de 156,000 personas en la industria creativa y cultural, lo que la convierte en la cuarta metrópoli europea en este tipo de producción, solo por detrás de París, Londres y Milán.

Llegados a este punto del análisis, comenzamos a entender la importancia que tiene la Economía creativa para el desarrollo y, sobre todo, nos aproximamos a comprender su potencial. Richard Florida (2010), desde una versión quizá un tanto optimista, ha llegado a identificar a la creatividad como la nueva economía. El autor sitúa como principal razón de crecimiento económico - destacablemente en los países desarrollados- a la creatividad humana. Una teoría que sustenta sobre tres ejes: el primero de ellos, que la creatividad es la primera fuente de riqueza en el mundo moderno, el segundo, que todo ser humano es creativo y, por último, que todos los trabajadores pueden poner en marcha su talento.

Fieles a las investigaciones de Florida, cabría pensar que podemos vislumbrar el alcance de la transformación que tenemos a la espera de ser puesta en marcha. Otras investigaciones ponen de manifiesto la importancia de la tecnología y el talento, así como de la fusión de las mismas como esencia para el crecimiento, y a ello, el autor le suma la idea de apertura de miras, en la diversidad y sobre todo en la tolerancia.

Por otro lado nos parece clave señalar el estudio sobre Economía creativa realizado para IPSOS, ${ }^{22}$ la tercera mayor empresa de investigación en el mundo y en el mercado de la inteligencia. En él, se resalta la idea de cómo la Economía creativa, quizá sin ser ideada y sin apenas base teórica ni políticas públicas que la potencien, tiene un crecimiento propiciado por la escasez que se da en los países más subdesarrollados. Ello significaría que a través de esa necesidad, el hombre estaría generando pequeños modelos de negocio

11 The European Cluster Observatory Priority Sector Report: Creative and Cultural Industries (2011).
12 Parte de los resultados se encuentran recogidos en el estudio realizado para IPSOS de la profesora Clo-tilde Pérez. Véase Pérez, 2015. 
ENGRANANDO LA FELICIDAD.

POLÍTICAS DE LA ECONOMÍA CREATIVA EN ESPAÑA

creativo, solo por supervivencia, sin apenas ser consciente de estar sumándose a la nueva tendencia de los territorios más desarrollados. De este modo cabría pensar que quizá son dos las vertientes desde las que nace el fenómeno creativo: desde la necesidad de crear para desarrollarse y desde el propio desarrollo para aproximarse a caminos sostenibles.

Así, desde esta doble perspectiva, lo que quizá nos resulte necesario entender, es que la creatividad está presente en el desarrollo de cualquier hombre, y como consecuencia, también de la sociedad en la que habita. Por tanto cobra relevancia la habilidad de desarrollar nuevas ideas y de mejorar las que ya existen. Afirman los autores Herrera-Medina, Bonilla-Estévez y Molina-Prieto que "los conglomerados de industrias -que se congregan en áreas específicas- impactan el desarrollo urbano, modelan la ciudad, le dan forma, generan polos de desarrollo y crean tensiones urbanas" $(2013,4)$. Además, señalan el proceso de materialización de la Economía creativa a través de nuevos formatos como los distritos culturales y creativos y los clusters.

Asimismo, podemos afirmar que la Economía creativa está mostrando, a través de diferentes vías, la gran repercusión que la creatividad tiene en el desarrollo económico de la sociedad de conocimiento, en la que ya nos encontramos, aunque sea desde diferentes niveles dependiendo del territorio donde se desarrolle.

Boix y Lazzeretti (2012) también resaltan la importancia de la creatividad en España situándola como la base de la innovación. Los autores insisten en lo necesario e importante que es el proceso de activación de la misma para obtener una fuente inagotable de mejora, desarrollo y competitividad, independientemente del sector al que se aplique. Nos guiamos así por los diferentes estudios analizados que afirman que la creatividad se ha convertido en un área crucial para la economía y que la innovación, además de ser extrapolable a cualquier sector de desarrollo, está resultando uno de los conceptos más asumidos en los últimos años. 


\section{Economía creativa y belleza}

¿Por qué cualquier producto o servicio creativo parece tener un mayor valor y estar directamente relacionado con el nivel de felicidad del ser humano que lo crea o desarrolla? Centrando nuevamente nuestra atención en el caso español, vemos que las áreas urbanas se enfrentan cada día al reto de elevar su capacidad competitiva, para así hacer frente a las devastadoras consecuencias derivadas de la crisis económica. ${ }^{13}$

Hemos señalado la necesidad de inventar nuevos modelos de desarrollo como uno de los orígenes de la aplicación creativa a la economía, y en esta misma frecuencia, parecen hallarse las actividades estratégicas que fomentan el desarrollo y comienzan a inyectar valor a una sociedad que aún no está del todo recuperada.

Observamos que de forma imparable aumenta el interés por las actividades ${ }^{14}$ integradas en la denominada Economía creativa, produciéndose un salto hacia el análisis de otros modelos y de la aplicación de la creatividad en sí misma, así como de su relación con otros sectores. Por el momento, podríamos pensar que una de las claves de activación de la Economía creativa en España podría estar en el buen aprovechamiento que los agentes económicos y administraciones hicieran de lo que se ha convertido en tendencia de desarrollo en otros países.

Aunque de otro lado, cabe preguntarnos: ¿sólo depende de los agentes económicos? Florida, en su visión optimista, propone ciudades que generen nuevas estructuras incluyentes y tolerantes, nuevas formas de organización social y nuevos entornos laborales que promuevan y estimulen la creatividad $(2010,12)$. Así, siguiendo la idea del autor, podríamos entender que quizá resulta relevante señalar a toda una sociedad como responsable del proceso de creación, es decir, po-

\footnotetext{
${ }^{13}$ Aludimos también a una crisis de valores sociales.
}

${ }^{14}$ En los últimos años son tendencia en muchas ciudades españolas la organización de encuentros y foros que analizan el concepto "creatividad" como fenómeno reciente. Un ejemplo de ello son algunas de las acciones que incluye el Ministerio de Educación, Cultura y Deporte. mecd.gob.es/cultura-mecd/areas-cultura/industriasculturales.html 
ENGRANANDO LA FELICIDAD.

POLÍTICAS DE LA ECONOMÍA CREATIVA EN ESPAÑA

dríamos pensar que el desarrollo de la Economía creativa de un territorio o ciudad y los beneficios de esta, son responsabilidad del ciudadano en sí, de la administración, de las universidades y de los tejidos empresariales del territorio, en general del trabajo colaborativo conjunto.

También hemos señalado el peso que están adquiriendo los resultados intangibles producidos por la conectividad que la Economía creativa comienza a generar en casi todos los sectores de desarrollo. A la sociedad consciente le interesan la sostenibilidad, la ecología, la cultura... de forma paralela aumenta la empatía, el respeto por el igual y la conciencia social pero ¿Qué tienen que ver estas tendencias con la Economía creativa? Mucho. Si pensamos en características propias de las mismas, veremos la relación directa. La Economía creativa parte de las industrias creativas ${ }^{15}$ y se expande a otros sectores generando bases de conciencia social, de autenticidad e innovación, de respeto, de sostenibilidad... en definitiva, de aspectos que generan un clima valioso en la comunidad.

Desde esta perspectiva, del acto de creación per se del hombre, obtenemos la esencia de la Economía creativa, una esencia que es capaz de crear e innovar, y no sólo en la industria cultural y creativa a la que podríamos denominar como el germen del fenómeno que analizamos, sino en la aplicación a cualquier sector.

Pensemos que el valor que puede proporcionar una idea puede ser muy superior al que proporcione un recurso natural sin procesar. ¿Podríamos entender entonces el aporte de belleza que el proceso creativo está generando en la sociedad? Pero, ¿Para qué sirve la belleza? Está demostrado que la belleza no impulsa el PIB de un país, pero ¿impulsa a los ciudadanos a una producción más creativa y productiva? Ordene (2013) valora muy positivamente la utilidad que tiene la belleza y, citando a Kant en su publicación (50), nos recuerda que la única satisfacción desinteresada y libre es la del gusto en lo bello, pues esta carece de interés alguno. Por lo tanto, cabe señalar la belleza como un todo que es suficiente en sí mismo y como tal, un aporte de valor para la sociedad. ¿Deben, en este sentido, dirigirse las políticas públicas a fomentar una corriente que genera la belleza que potencia la crea-

\footnotetext{
15 En el informe de La UNCTAD se consideran las industrias creativas como el corazón de la economía creativa, $(2010,37)$.
} 
ENGRANANDO LA FELICIDAD.

POLÍTICAS DE LA ECONOMÍA CREATIVA EN ESPAÑA

tividad del hombre? A priori, puede resultar una idea sin fundamento, pero en realidad, se trata de un fenómeno muy hondo que comienza a ser observado minuciosamente. ${ }^{16}$

Una razón que también da sentido a esta teoría, podría ser la que aportan los autores Méndez, Michelini, Prada y Tébar (2012) cuando señalan el proceso de cómo se desliga la economía del sesgo tecnológico para ser incluida en actividades de un alto contenido simbólico y donde los recursos intangibles generan un valor añadido, resaltando como ejemplo de ello a las actividades culturales. Se trata por tanto, de un importante proceso que parece humanizar al concepto de economía.

Hechos como los anteriores, están suponiendo generación de empleo, cooperación ciudadana en el desarrollo local, remodelación de la imagen urbana, asociacionismo, la suma en acción de diversos perfiles profesionales... en definitiva, sólidas estructuras que parecen estar dando sentido a la tímida regeneración de políticas públicas así como a las nuevas estrategias de revitalización de territorios.

Un ejemplo de ello son los resultados obtenidos de la observación de otras ciudades, que han conseguido evolucionar en direcciones alternativas a partir de los modelos que provienen del fenómeno creativo. En el caso de Islandia, la clave para la administración del país han sido las personas y también apostar por la industria cultural y creativa. ${ }^{17}$ El país ha dirigido sus políticas públicas hacia el objetivo de recuperación de la confianza. La democracia se ha convertido en una fuerza económica poderosa, siendo el éxito de la economía la base de fomentar la creatividad de las personas. Y es que cabe preguntarnos: ¿acaso el sector financiero es mejor para el buen estado y el futuro de la economía que el creativo?

\footnotetext{
${ }^{16}$ Nuccio Ordine en su publicación La utilidad de lo inútil hace especial hincapié en la importancia que ha tenido y tiene la belleza para el hombre.
}

\footnotetext{
17 Islandia se ha volcado en el sector de las industrias creativas. Hoy la tasa de paro islandesa es del 5,7\% y el país crece a un ritmo del $3 \%$. No es casualidad que los sectores de la cultura y la creatividad islandeses empleen a más del $5 \%$ de la fuerza laboral, más que las industrias pesquera y agrícola juntas, y además generen un porcentaje del PIB superior al de la agricultura. Véase: repensadores.es/2013/11/porque-es-tan-importante-la-industria-cultural-y-creativa-paracualquier-pais/
} 


\section{Creatividad y políticas públicas en España}

Hemos visto cómo la creatividad brota con fuerza en el ser humano y adquiere sentido a través de cada proceso. De modo intuitivo, el concepto creatividad nos remite a la capacidad no sólo de crear lo nuevo, sino también de reinventar, de diluir prototipos tradicionales, de unir puntos aparentemente inconexos y, con ello, activar la capacidad de plantear soluciones de desarrollo.

En la misma dirección, hemos comprobado como dentro del sector económico, la creatividad se está convirtiendo en combustible renovable, que parece incrementarse con su uso. Y, desde esta lógica, podríamos pensar que por el momento, no existe mejor herramienta que la creatividad para regenerar las políticas públicas de un territorio. No sólo se trata de un fenómeno de la última década, pues aunque ahora éste tenga más fuerza y se vaya consolidando a través de una reformulación del papel de la cultura y la creatividad en los entornos urbanos, ya en la década los años noventa, Hall (1996) resaltaba su gran protagonismo económico cada vez más reconocido; aunque en realidad, se trata de una visión que comenzó con el proyecto Creative Nation celebrado en Australia en 1994 donde se destacaba el aporte económico de las actividades creativas de ese país (Herrera-Medina, 2013,4).

Desde una visión más actual y ya abordando el desarrollo de políticas públicas, durante una de las ponencias que formaron parte de las jornadas de Creaurbs $^{18}$ se hacía hincapié en la idea de que la regeneración de políticas públicas en este ámbito pudo haber estado marcada -principalmente- por dos variables que la condicionaron: la gobernanza multinivel y el desarrollo histórico de cada territorio.

\footnotetext{
18 CREAURBS: Creatividad y conocimiento: Bases para una nueva competitividad urbana realizadas el 13 y 14 de diciembre de 2012 en la Facultad de Economía y Empresa de la Universidad de Barcelona.
} 
ENGRANANDO LA FELICIDAD.

POLÍTICAS DE LA ECONOMÍA CREATIVA EN ESPAÑA

Algunas de las conclusiones de las ponencias apuntaban hacia la idea de que las principales intervenciones de esta nueva visión de la cultura y de la creatividad parecieron estar orientadas, en su mayoría, a estrategias de regeneración urbana y fomento de las nuevas economías urbanas, al desarrollo y promoción de agentes, empresas y colectivos y a la estimulación de la una cultura social innovadora y creativa que favorezca la aparición de ideas y proyectos innovadores, entre otras.

Lo cual nos resulta un dato interesante para averiguar la tendencia que va adquiriendo la retórica que conforma las políticas públicas.

\section{Políticas públicas de desarrollo en las principales ciudades españolas}

Con la finalidad de señalar las políticas públicas actuales enfocadas a las industrias creativas, nos hemos remontándonos a años atrás mediante algunos de los resultados expuestos en las jornadas de Creaurbs que han medido elementos comunes de desarrollo y crecimiento de cuatro de las principales ciudades españolas: Barcelona, Bilbao, Madrid y Valencia.

En los años 50 y 60 del pasado siglo, las industrias se producían fuera de los centros urbanos, así como también un proceso de consolidación de áreas metropolitanas más o menos centralizadas. Años más tarde, durante la transición española, a la vez que nacían los gobiernos autonómicos con cierta tendencia tecnocrática, se producía un momento de recesión de la producción industrial (Pradel 2012). De este modo, podríamos pensar que ambas razones pudieron hacer que nuevas políticas públicas persiguieran el objetivo de regenerar los centros urbanos mediante la aplicación de la innovación a la industria. 
ENGRANANDO LA FELICIDAD.

POLÍTICAS DE LA ECONOMÍA CREATIVA EN ESPAÑA

Ante la problemática expuesta, la aparición de la fuerte crisis de las ciudades industrializadas, hizo que emergieran con fuerza discursos y perspectivas que planteaban la necesidad de una reestructuración y reactivación económica de los modelos productivos en estas áreas industrializadas y que, a su vez, potenciaran la transformación y regeneración de los espacios urbanos y regionales. Continuando en esta dirección, ya en los años noventa, con la finalidad de potenciar el crecimiento de los centros urbanos y siguiendo la tendencia europea, comenzaría a ser muy necesaria la renovación de las políticas de desarrollo local, los objetivos de la regeneración urbana como motor de desarrollo eran, principalmente, los de atraer visitantes a través del turismo de negocios y de ocio, de generar entornos innovadores que atrajeran al tejido empresarial y mejoraran las infraestructuras, y como resultado de estos procesos, que aumentara la calidad de vida.

Podríamos pensar que la finalidad última de incorporar la creatividad en los planes de desarrollo no sería otra en definitiva que la de crear entornos atractivos en los que el ciudadano deseara trabajar, vivir y visitar.

¿Por qué apostar por la creatividad para mejorar las políticas públicas? Hoy, para muchos autores comienza a ser una de las opciones más válidas a tener en cuenta (Fonseca, 2008).

Parece ser que el gran diferencial de la Economía creativa con otros modelos, no es otro que promover un desarrollo sustentable y humano, no quedándose únicamente en el plano del beneficio económico, sino como un acto incluyente social, hace que el ser humano forme parte siendo -incluso- el motor del proceso de desarrollo. Un proceso natural de crecimiento que existió en España y que se vio paralizado por la adaptación de las políticas públicas hacia el sector de la construcción como eje principal de desarrollo. Durante la década del año 2000, esta puesta en marcha de las políticas de regeneración urbana se fragmentó y fue paralizada por la sobreexplotación de lo que se consideraría una potencia para generar riqueza. La construcción de entornos "ideales" se consideró como uno de los principales motores para el desarrollo, olvidándose de la tendencia anterior de crear la ciudad postindustrial, un ciudad bella, limpia, vanguardista, multicultural, creativa, inteligente, sostenible...(Rodríguez y Vicario, 2005). 
ENGRANANDO LA FELICIDAD.

POLÍTICAS DE LA ECONOMÍA CREATIVA EN ESPAÑA

Posterior a este proceso, las políticas de promoción económica comenzaron a apuntar hacia la creación de planes de clusterización, apostando para ello por el desarrollo de otros sectores que pudieran resultar estratégicos. También se reforzaron las políticas de innovación, se crearon parques tecnológicos, para fomentar la vinculación de la empresa con la universidad. En esta línea, también se hizo hincapié en el desarrollo de políticas de capital humano, creándose numerosas agencias de desarrollo local que potenciaran el emprendimiento y la formación especializada.

Según los resultados de las jornadas de Creaurbs en 2012, las estrategias de desarrollo económico aplicadas en Barcelona estuvieron enfocadas a impulsar las industrias culturales en el centro de la ciudad, así como la economía de conocimiento a través de la regeneración urbana. También parece que la ciudad catalana comenzó a crear Agencias de Desarrollo Local. En cuanto a la ciudad de Bilbao, se identificó un gran impulso de la industria del conocimiento, intentando a la vez generar un equilibrio con el tejido industrial ya existente y, en la misma dirección, la regeneración urbana pareció convertirse en un potente motor de la economía de la capital. Una de las características más importantes dentro de las políticas públicas en la capital española fue, sin duda, el liderazgo del sector privado y la participación del sector público. Madrid tendió a impulsar las industrias culturales a través de las grandes instituciones culturales y de la regeneración urbana, prestando atención en políticas de capital humano y de emprendimiento. La última ciudad analizada, Valencia, centró sus políticas públicas en la promoción de la ciudad, con la final intención de convertirla en centro de turismo y negocios, siendo escasas las políticas públicas enfocadas a la innovación.

A través de este breve recorrido por los discursos de creación de políticas públicas en los últimos años en España, podemos apuntar las siguientes conclusiones:

- Parece existir en el territorio español la tendencia de fomentar el conocimiento.

- La creatividad y la producción artístico-cultural comienza a jugar un nuevo papel en la promoción de la innovación social y en los entornos socialmente creativos, en la medida en que son capaces de movilizar recursos y capacidades en los entornos locales, convirtiéndose en desarrollo territorial. 
ENGRANANDO LA FELICIDAD.

POLÍTICAS DE LA ECONOMÍA CREATIVA EN ESPAÑA

- La transferencia del conocimiento, las sinergias, los espacios de trabajo conjunto y las colaboraciones público-privadas, son algunas de las acciones que se comienzan a llevar a cabo para conseguir un desarrollo más eficiente en los espacios urbanos españoles, especialmente en las ciudades de mayor tamaño (Madrid y Barcelona).

- Se reconducen las políticas públicas de desarrollo hacia la regeneración urbana mediante la potenciación de sectores como las industrias culturales y creativas.

- Parecen existir crecientes evidencias en el territorio español de que el sector cultural y creativo moviliza, estimula y atrae recursos favorecedores para el desarrollo urbano. ${ }^{19}$

- La generación de políticas de ganancia parece quedar atrás, dando lugar a nuevos métodos de crecimiento más lentos y seguros.

- Puede que algunos de los intereses de los sectores consolidados se vean enfrentados con los objetivos de las nuevas políticas de desarrollo por lo que podría hacerse necesaria la estructuración de un plan que fomente la convivencia de ambas.

- Los actores locales parecen -poco a poco- familiarizarse en la adopción de los discursos de crecimiento, aunque en la mayoría de casos, se ha comprobado un proceso de escasa innovación institucional. Un ejemplo de ello serían los Ayuntamientos de algunas ciudades españolas como Murcia que comienzan a incluir en sus discursos conceptos próximos a la economía creativa (participación ciudadana, asociacionismo...) pero sus políticas aún no apoyan de modo directo la industria creativa.

\footnotetext{
19 Sirva de ejemplo La Red de industrias creativas que se nutre de empresas, profesionales e instituciones de diferentes países: México, Venezuela, Costa Rica, Chile, Colombia, Italia, Francia y España. Se trata de una red de alianzas comprometidas con el desarrollo de la industria creativa en español.
} 
ENGRANANDO LA FELICIDAD.

POLÍTICAS DE LA ECONOMÍA CREATIVA EN ESPAÑA

- Algunos de los problemas de la centralización autonómica vienen de la escasa autonomía local, del desconocimiento de las escalas de desarrollo adecuado, de la falta de resultados en cuanto a si los mecanismos de mercado fomentan el desarrollo local (CREAURBS, 2012).

De este modo, teniendo en cuenta que no existe un política nacional integrada para las industrias creativas, sólo planes como El Plan para la promoción de las industrias culturales (2015), enfocado en su mayoría a fomentar la parte más inicial de las industrias creativas (sector cultural), y siguiendo la tendencia analizada de cooperatividad, originalidad y búsqueda de esencia de los territorios, deberíamos pensar en lo beneficioso que sería para España un plan nacional de desarrollo local sustentado en bases de Economía creativa, ideado desde un trabajo de colaboración público- privada a través de procesos de participación ciudadana.

Como hemos visto, algunos gobiernos regionales de ciudades españolas han adoptado dentro de sus políticas urbanas conceptos surgidos de la Economía creativa, como por ejemplo el caso de Sevilla con el concepto "distrito cultural" o Málaga que, mediante una colaboración público privada genera un espacio de encuentro para apoyar los planes de emprendimiento cultural. ${ }^{20}$ A su vez, Bilbao fue incorporada a finales de 2014 a la Red de ciudades creativas de la UNESCO como "ciudad creativa".

Estas serían algunas de las acciones más significativas que se están dando en el territorio español próximas al discurso de Economía creativa , lo cual nos demuestra que aún queda trabajo por la inclusión del término en los programas de desarrollo, en los programas académicos de las universidades, ${ }^{21}$ desde las administraciones...

Otras acciones destacables de reciente creación, son:

El Foro Europeo de la Cultura organizado por la Comisión Europea, con el fin de poner de relieve el papel de la cultura como un caldo de cultivo para el talento y la creatividad, el 20 Para el caso de Sevilla véase icas-sevilla.org/ para el caso de
Málaga véase locosporlacultura.com/
21 Sólo hemos identificado un programa de formación sobre Economía creativa asociado a la Universidad Rey Juan Carlos I en la capital española: Máster en Economía Creativa mastereconomiacreativa.es/ 
ENGRANANDO LA FELICIDAD.

POLÍTICAS DE LA ECONOMÍA CREATIVA EN ESPAÑA

I Encuentro de Espacios Creativos Culturales, Zinc Shower, Think Up Culture y EuropeanCreativeHubsForum, son encuentros organizados con la colaboración de la Secretaría de Estado de Cultura por iniciativas privadas. También la fundación INCYDE, en colaboración con la Secretaría de Estado de Cultura, ha organizado jornadas sobre instrumentos de financiación a disposición del sector de las ICCs.

Con programas de este tipo, vemos que la colaboración público-privada es activa en algunas ciudades españolas, haciendo que proyectos que se iniciaron de forma privada, adquieran relevancia y se posicionen como herramientas de medición y activación de Economía creativa.

\section{Economía creativa: desarrollo de ciudades y del ciudadano}

¿Por qué enfatizar la Economía creativa?

1 Crea ecosistemas desde creatividad humana.

2 Genera valor económico.

3 Convierte al territorio en pieza clave para crear entornos felices.

Ana Carla Fonseca, una de las mayores expertas en el estudio de Economía creativa afirmaba:

En los últimos diez años pocos conceptos han sido más debatidos, menos definidos y tan poco considerados de modo filtrado, traducido e reinterpretado para países con contextos culturales, sociales y económicos distintos, en una miríada de vertientes: ciudades creativas, industrias creativas, economía creativa, grupos creativos, clase creativa, activos creativos $(2008,11-12)$.

Como veíamos con anterioridad, La UNESCO, en 2006; UNCTAD, en 2008 y 2010; y la Comisión Europea, en 2010, han sido algunas de las instituciones internacionales, que han otorga- 
ENGRANANDO LA FELICIDAD.

POLÍTICAS DE LA ECONOMÍA CREATIVA EN ESPAÑA

do mayor importancia a la creatividad, entendida, sobre todo, desde su capacidad de aportar respuestas nuevas y eficaces frente a los retos a los que se enfrentan los individuos, las sociedades, los territorios..., convirtiéndose, cada vez más, en un concepto cercano y frecuente.

También hemos observado que la progresiva mercantilización de la creatividad, aplicada a cada área, está generando en el mercado una importante oferta de bienes de simbólico contenido, haciendo que las políticas públicas presten más atención a los resultados. De este modo, comprobamos que los recursos utilizados por los sectores creativos, la mayoría intangibles, comienzan a concentrarse en determinadas ciudades con características tan específicas como la construcción social, convirtiéndose en objetos de creciente atención de las políticas urbanas, tanto por su efecto potencial de revitalización económica como por la capacidad de creación de nuevas imágenes de ciudad.

Cabe señalar que una de las consecuencias negativas de la indefinición de la Economía creativa (hemos visto que se trata de un concepto recientemente joven), sea la falta de acuerdo en la relación de actividades que deban incluirse dentro del nuevo modelo, y como consecuencia, que afecte a la adaptación de las políticas públicas que sirvan para potenciarlas. Pero, independientemente a las diferentes clasificaciones de las actividades que pertenecen a la Economía creativa establecidas por los diferentes organismos internacionales, que poco a poco se irán posicionando, resulta interesante resaltar, además de las lógicas razones culturales a favor de este sector, una serie de argumentos económicos que parecen dar más sentido a la aplicación de la Economía creativa en la creación de políticas públicas.

Con los estudios analizados para este artículo y con el análisis de las tendencias del territorio español respecto a la aplicación de la Economía creativa, podríamos pensar optimistamente que nos aproximamos a un desarrollo urbano más lento, pero más seguro, vislumbrando en esta dirección, un gran potencial de crecimiento para la próxima década.

Por lo tanto, si pensamos en ejes transversales de actuación, deberíamos apuntar hacia esa fusión de conceptos innovadores sobre los que ya parece asentarse el fenómeno creativo, a enfocar al desarrollo local de los territorios y a contar con expertos que ayuden a mejorar algunas de las retóricas que han podido quedar obsoletas para el desarrollo de políticas públicas de crecimiento. Otro de los asuntos que impulsaría el potencial creativo de los ciudadanos y de los territorios 
ENGRANANDO LA FELICIDAD.

POLÍTICAS DE LA ECONOMÍA CREATIVA EN ESPAÑA

sería el desarrollo de formaciones específicas en el campo de la Economía creativa. Sobre esta idea, Richard Florida (2010) considera de gran valor el hecho de invertir nuevamente en el acto de potenciar las universidades, ya que según el autor, son imanes de talento y de tolerancia, defendiendo la idea de que dichas instituciones deberían ser convertidas en el foco de la Economía creativa.

Nos posicionamos en la idea de que será tan sólo el factor tiempo el que dé lugar al nacimiento de nuevos métodos educacionales y programas formativos centrados en la aplicación de la creatividad en todos y cada uno de los perfiles profesionales, innovando así en las bases de un sistema educativo que parece estar anclado en la época industrial y que debería reflejar y reforzar los valores de los nuevos tiempos que exigen, más que nunca, una formación integral (Florida, 2010).

En esta dirección, hoy observamos desde una atenta mirada a un sector que, hasta ahora, sólo ha aportado magníficas noticias a la economía mundial, a la creación de empleo, al cambio de modelo económico, al desarrollo... Aunque quizá, uno de los aspectos más importantes a tener en cuenta sea el gran aporte que hace al ser humano, a su educación y a su crecimiento, y como consecuencia de ello, a su entorno.

¿Podríamos pensar que, lentamente, la humanidad, con un gesto de creatividad está comenzando a aprovechar la ocasión para ser más humana? Quizá lo más interesante del fenómeno creativo no sólo sea que parece tener la llave del crecimiento económico, sino que, además, pueda impulsar el desarrollo del potencial humano en general.

Finalizamos este artículo con una cita del famoso pedagogo estadounidense Abraham Flexner recogida en La utilidad de lo inútil de Nuccio Ordine:

Por nuestra parte, no prometemos nada, pero abrigamos la esperanza de que la libre búsqueda de conocimientos inútiles demostrará tener consecuencias en el futuro como las ha tenido en el pasado. Ni por un momento, sin embargo, defendemos el Instituto por esta razón. Existe como un paraíso para los estudiosos que, como los poetas y los músicos, se han ganado el derecho a hacer las cosas a su gusto y logran los mayores resultados cuando se les permite actuar así $(2013,172)$. 
ENGRANANDO LA FELICIDAD.

POLÍTICAS DE LA ECONOMÍA CREATIVA EN ESPAÑA

\section{Referencias}

Adorno, T. (1967). La industria cultural, en Morin, E. \& Adorno, T., La industria cultural, Buenos Aires: Galerna, pp. 7- 20.

Aguiar, E. (2014). Las industrias culturales y creativas en España. Cátedra UAM-Accenture en Economía y Gestión de la Innovación. Recuperado de uam.es/ docencia/degin/catedra/documentos/13_Las\%20 industrias $\% 20$ culturales $\% 20 y \% 20$ creativas $\% 20$ en\%20Espana.pdf

Precedo, A. J. (1993). Las políticas de desarrollo y renovación urbana de Europa, Ciudad y territorio: estudios territoriales, (98), pp. 579-95.

Balbo, M.; Jordán, R. y Simioni, D. (Comp.) (2003). La ciudad inclusiva Cuadernos de la CEPAL, (88). Recuperado de eclac.org/publicaciones/ xml/7/14237/lcg2210p.pdf

Borja, J. (2010). Luces y sombras del urbanismo de Barcelona., UOC (Ed.), Colección Gestión de la Ciudad. Recuperado de upcommons.upc.edu/eprints/bitstream/2117/12415/1/5488763.pd

Boix, R. \& Lazzaretti, L. (2012). Las industrias culturales en España: una panorámica en Investigaciones Regionales. (22). pp.181- 206

Buitrago, F. \& Duque, I. (2013). La economía naranja: una oportunidad infinita. Banco Interamericano de Desarrollo.

Cañedo, M. (2006). Lavapiés, área de rehabilitación preferente. Políticas culturales y construcción del lugar. Madrid: UCM [tesis inédita]

Hall, P. (1996). Ciudades del mañana: historia del urbanismo en el siglo XX, Madrid: Ediciones del Serbal.
(2012). Economía política y sentido de la creatividad en la ciudad contemporánea: los espacios públicos del multiculturalismo en Madrid. Bilbao. Jornadas Antropología Urbana Eusko Ikaskuntza. Recuperado de espacio.uned.es/fez/ eserv/bibliuned:500384-1010/Documento.pdf

Comisión Europea, Libro Verde, (2010). Liberar el potencial de las industrias culturales y creativas. Bruselas: Autor. Recuperado de eurlex.europa.eu/LexUriServ/LexUriServ. do?uri=COM:2010:0183:FIN:ES:PDF

Echevarría, J. (2013). El debate sobre las industrias culturales y creativas, Cuadernos hispanoamericanos, (761), pp. 21-33.

Fonseca Reis, A. (Comp.) (2008). Economía creativa: como estrategia de desarrollo: una visión de los países en desarrollo, Sao Paolo: Itaú Cutural. Recuperado de sic.conaculta.gob.mx/centrodoc_ documentos/555.pdf

Florida, R. (2010). La clase creativa: la transformación de la cultura del trabajo y el ocio en el Siglo XXI. Barcelona: Paidós Ibérica.

Galarraga, A.; González, S. \& Luna, A. (2013). Cultura y creatividad en la nueva economía urbana: evidencias, discursos y críticas en Revista de Dirección y Administración de Empresas, (20), pp. 33-55, Universidad del País Vasco.

Garnham, N. (2011). De las industrias culturales a las creativas. Análisis de las implicaciones en el Reino Unido en Bustamante, E. (2011). Industrias Creativas. Amenazas sobre la cultura digital. Barcelona: Gedisa. 
ENGRANANDO LA FELICIDAD.

POLÍTICAS DE LA ECONOMÍA CREATIVA EN ESPAÑA

O’connor R, J. (2011). Las industrias culturales y creativas: una historia crítica, en Ekonomiaz: revista vasca de economía, (78), pp. 24-47.

Ascher, F. (2007). Los nuevos principios del urbanismo. Madrid: Alianza

González, S. (2011). Bilbao and Barcelona 'in motion': how urban regeneration 'models' travel and mutate in the global flows of policy tourism, Urban studies. Recuperado de usj.sagepub.com/ content/early/2010/09/09/0042098010374510

Howkins, J. (2013). The creative economy: how people make money from ideas. $3^{\mathrm{a}} \mathrm{Ed}$, London: Penguin Books.

Manito, F. (Ed.). (2010). Ciudades creativas.

Vol. 2: Creatividad, innovación, cultura y agenda local. Barcelona: Bellaterra Ediciones

Méndez, R.; Michelini, J.J.; Prada, J. \& Tébar, J. (2012). Economía creativa y desarrollo urbano en España: una aproximación a sus lógicas espaciales. Revista EURE - Revista Latinoamericana de Estudios Urbano Regionales. Recuperado de scielo.cl/pdf/eure/ v38n113/art01.pdf

Ordine, N. (2013). La utilidad de lo inútil. Manifiesto. $11^{\text {a }} \mathrm{Ed}$, Barcelona: Acantilado.

Pérez, C. (2015). La economía creativa como tendencia social en Estudio mundial de tendencias del Instituto IPSOS. Universidad de Murcia.

Throsby, D. (2001). Economía y Cultura. Madrid: Cambridge University Press

UNESCO Organización de las Naciones Unidas para la Educación, la Ciencia y la Cultura (2006). Understanding creative industries: Cultural statistics for public-policy making. Paris: UNESCO.
Rius, J. (2008). Los barrios artísticos como base local de la cultura global: el caso del Raval de Barcelona, Revista internacional de sociología, (51), pp. 179-205.

Rodríguez, A. y Vicario L. (2005). Innovación, competitividad y regeneración urbana: los espacios retóricos de la ciudad creativa en el nuevo Bilbao, en Ekonomiaz: revista vasca de economía, (58), pp. 262-295. Recuperado deeuskadi.net/ekonomiaz/ taula4_c.apl?REG=740

Pradel, M. (2012). Gobernanza y politicas públicas para la economía creativa y del conocimiento en España, en CREAURBS. Creatividad y conocimiento: Bases para una nueva competitividad urbana. Facultad de Economía y Empresa de la Universidad de Barcelona. Recuperado de: ub.edu/ubtv/es/colleccio/ congressos-i-jornades/creatividad-y-conocimientobases-para-una-nueva-competitividad-urbana

Sossa, A. (2010). La alienación en Marx: el cuerpo como dimensión de utilidad en Revista Ciencias Sociales, (25), pp.37-55. Recuperado derevistacienciasociales. cl/archivos/revista25/pdf/rcs-art3.pdf

Throsby, D. (2008). From cultural to creative industries: The specific characteristics of the creative industries. fil Journées d'Économie de la Culture

UNCTAD Conferencia de las Naciones Unidas sobre Comercio y Desarrollo. (2010). Creative Economy Report. Ginebra-Nueva York: UNCTAD.

UNESCO Organización de las Naciones Unidas para la Educación, la Ciencia y la Cultura. (2010). Políticas para la creatividad. Guía para el desarrollo de las industrias culturales y creativas. 